\title{
Study of nonlinear convection in a sparsely packed porous medium using spectral analysis
}

\author{
N. RUDRAIAH and S. BALACHANDRA RAO
}

UGC-DSA Centre in Fluid Mechanics, Department of Mathematics, Central College, Bangalore University, Bangalore-560001, India

\begin{abstract}
Nonlinear study cellular convection in a sparsely packed fluid saturated porous medium is investigated, considering the Brinkman model, using the technique of spectral analysis. It is established how the Brinkman model with free-free boundaries generalizes the study of convection in a porous medium in the sense that it yields the results tending to those of viscous and Darcy flows respectively for very small and very large values of the permeability parameter $\sigma^{2}$. It also provides results for the transition zone (i.e. $10^{2}<\sigma^{2}<10^{3}$ ). The cross-interaction of the linear modes caused by non-linear effects are considered through the modal Rayleigh number $\mathrm{R}_{\gamma}$. The possibility of the existence of steady solution with two self-excited modes in certain regions is predicted. The similarities of present analysis with and advantages over that of the power integral technique are brought out. A detailed discussion of the heat transport, with the effect of permeability thereon, is made. The theoretical values of the Nusselt number are found to be in good agreement with experimental results.
\end{abstract}

\section{Introduction}

In recent years, considerable interest has been evinced in the study of thermal convection in fluid saturated horizontal porous layer because of its intrinsic importance and its relevance in nature as well as in technology. This problem is usually studied $[6,7,10,13,14,16,21,24]$ using the Darcy model which results in a fourth-order differential equation to govern the onset of instability. Using physical arguments, however, six boundary conditions based on no-slip can be specified (see [20,21]). In a mathematical sense the problem is therefore ill-posed and we have an overspecified system. From a physical point of view, the no-slip condition [12] is as much valid as the other two conditions and there appears a priori no reason to reject it. Therefore, the applicability of Darcy's Law to free convective studies in a porous medium appears to be questionable and a fresh approach to this problem is warranted. A suitable statistical approach is needed to resolve this problem. If a porous medium, how- 
ever, is made up of sparse distribution of solid particles, Tam [22], Brenner [2], Taylor [23], and Rudraiah and Veerabhadraiah [17,18,19] have shown that the Brinkman [3] model comprising both viscous shear and Darcy resistance is valid to a first approximation. This Brinkman model is of boundary layer type and gives rise to a sixth-order differential equation with six boundary conditions for the study of convection in a porous medium. Care must be taken in using these no-slip boundary conditions in the case of a porous medium. If it is bounded by rigid impermeable plates, one has to apply correction to the no-slip condition analogous to the slip condition postulated by Beavers and Joseph [1]. Such difficulties, however, will not arise in the case of a porous medium bounded by free-free boundaries where slip is allowed. Therefore, in this paper the nonlinear convection in a fluid saturated porous medium is studied using the Brinkman model with free-free boundaries. The novelty of using this model lies in: (i) overcoming the mathematical difficulty posed by Darcy's model as explained above, (ii) generalizing the problem in the sense that for small and large values of the permeability parameter $\sigma^{2}$ the results tend respectively to those of the viscous and the Darcy flows in addition to providing results in the transition zone [15] for finite values of $\sigma^{2}$, and (iii) predicting the magnitude of heat transfer after the onset of convection in a fluid saturated porous layer as accurately as possible.

The study of finite amplitude convection in a porous layer is usually based on using the power integral technique which is pivoted on the linear theory. This is an iterative technique which combines the best features of the Galerkin method and Stuart's shape assumption, first employed by Malkus and Veronis [11]. This power integral technique is mathematically cumbersome and the built-in orthogonality process to overcome the resonance and the secural terms takes into account only the even modes. In the process, some of the interesting results, obtained by considering cross-interactions of modes, are missed. In the case of Darcy flow, recently Rudraiah and Balachandra Rao [14] have overcome this deficiency using the method of spectral analysis as adopted by Kuo and Platzman [9]. In the present paper, we therefore use this spectral analysis to study the onset of convection in a fluid saturated porous medium using the Brinkman model.

The interesting results obtained in this paper using the Brinkman model are delineated by comparing these with the existing results for purely viscous flow [9] and for the Darcy flow [14].

\section{Mathematical formulation}

The physical configuration considered in this paper (see Fig. 1) consists of a thin horizontal porous layer, of thickness $d$, of infinite extent in the horizontal directions heated uniformly from below and cooled from 
above. The temperature difference across the layer is $\Delta T=T_{1}-T_{2}(>0)$, the lower boundary being at temperature $T_{1}$ and the upper one at $T_{2}$.

The equations governing convection in a porous layer, following Rudraiah et al. [20], are

$$
\begin{aligned}
& \frac{\partial \boldsymbol{q}}{\partial t}+(\boldsymbol{q} \cdot \nabla) \boldsymbol{q}=-\frac{1}{\boldsymbol{\rho}_{0}} \nabla p+\alpha g T \hat{k}+\nu \nabla^{2} \boldsymbol{q}-\frac{\nu}{k} \boldsymbol{q} \\
& (\rho C) * \frac{\partial T}{\partial t}+(\rho C)_{f}(\boldsymbol{q} \cdot \nabla) T=K^{*} \nabla^{2} T \\
& \nabla \cdot \boldsymbol{q}=0 \\
& \rho=\rho_{0}\left[1-\alpha\left(T-T^{\prime}\right)\right]
\end{aligned}
$$

where

$$
(\rho C)^{*}=\varepsilon(\rho C)_{f}+(1-\varepsilon)(\rho C)_{s},
$$

$K^{*}$ is the effective thermal conductivity of the porous medium, $p$ is the pressure in excess of the hydrostatic value, $\rho$ is the fluid density and $\rho_{0}$ is that at the ambient temperature $T^{\prime}, T$ is the fluid temperature, $\boldsymbol{q}=$ $(u, v, w)$ is the mean filter velocity, $\hat{k}$ is the unit-vector in the $z$-direction, $(\rho C)^{*}$ is the relative heat capacity of the porous medium while $(\rho C)_{f}$ and $(\rho C)_{s}$ are those of fluid and solid respectively, $\varepsilon$ is the porosity and $k$ is the permeability, having the dimension of length squared, for the porous medium, $\alpha$ is the thermal expansivity of the fluid, $g$ is the acceleration due to gravity and $\nu$ is the kinematic viscosity of the fluid.

Recently, Rudraiah and Srimani [16] have shown, considering the Darcy model, that two-dimensional rolls are the preferred cell pattern in the case of convection in a porous medium. Hence, in the present paper cellular convection consisting of two-dimensional rolls is considered. The marginal state is assumed to be valid since there is no external constraint like rotation, magnetic field or salinity gradient to make the velocity and temperature out of phase. Therefore, a steady solution of the nonlinear stability of a quiescent state will be obtained by superposing a symmetric perturbation such that $\partial / \partial y=0$ and $\partial / \partial t=0$.

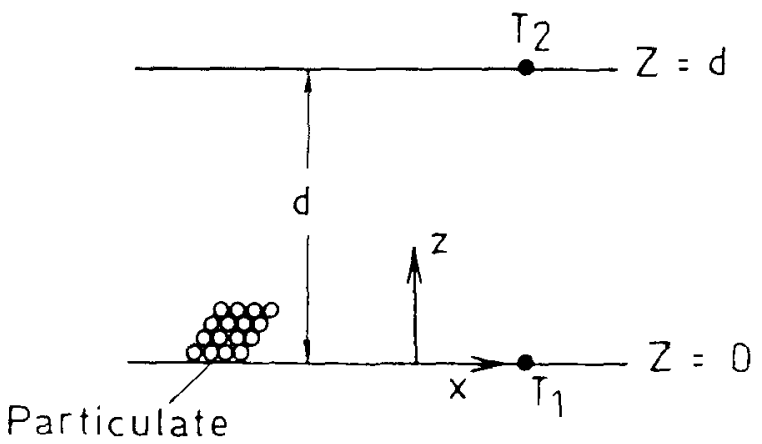

Figure 1. Schematic illustration of a porous layer 
Using the total non-dimensional temperature $T$, given by

$$
T=\left(T_{1}-R z\right)+\theta
$$

the stream function $\psi$ such that

$$
u=-\frac{\partial \psi}{\partial z}, \quad v=\frac{\partial \psi}{\partial x},
$$

eliminating the pressure in Eqn. (2.1) and making the resulting equations dimensionless using

$$
d / \pi, \quad d^{2} / \pi^{2} \kappa^{*}, \quad \pi \kappa^{*} / d \quad \text { and } \quad \pi^{2} \kappa^{*} \nu / \alpha g d^{3}
$$

as the units of length, time, velocity and temperature respectively, we obtain the following steady state vorticity equation

$$
\left(\nabla^{2}-\sigma_{1}^{2}\right) \nabla^{2} \psi+\frac{\partial \theta}{\partial x}=\frac{1}{\operatorname{Pr}} B
$$

where $B=\left[\partial\left(\psi, \nabla^{2} \psi\right)\right] /[\partial(x, z)]$ is the vorticity advection Jacobian, $\operatorname{Pr}$ $=\nu / \kappa^{*}$ is the Prandtl number, $\sigma_{1}^{2}=d^{2} / \pi^{2} k$ is the modified permeability parameter. In fact, $\sigma_{1}^{2}=\sigma^{2} / \pi^{2}$ where $\sigma^{2}=d^{2} / k$ is the usual permeability parameter. Note that $\pi$ appears in all these terms because of our choice of the length scale.

The non-dimensional steady state energy equation is

$$
\nabla^{2} \theta+R \frac{\partial \psi}{\partial x}=H
$$

where $H=[\partial(\psi, \theta) / \partial(x, z)]$ is the thermal advection jacobian and $\mathrm{R}=$ $\alpha g \Delta T d^{3} / \pi^{3} \nu \kappa^{*}$ is the Rayleigh number. This differs from the Lapwood [10] Rayleigh number, $\mathrm{R}_{L}=\alpha g \Delta T d k / \nu \kappa^{*} \pi$, by a factor $1 / \sigma_{1}^{2}$, i.e. $\mathrm{R}_{L}=$ $R / \sigma_{1}^{2}$.

The use of no-slip boundary condition poses a problem in the case of a porous medium bounded by rigid impermeable plates. In that case one has to apply a correction to the no-slip condition analogous to the one given by Beavers and Joseph [1]. However, such difficulties will not arise in the case of a porous medium bounded by free-free boundaries where, of course, slip is allowed and there is no need to apply a correction for no-slip. Thus, the required boundary condition, since we consider the stress-free isothermal boundaries, are

$$
\psi=\nabla^{2} \psi=\theta=0 \quad \text { at } \quad z=0,1
$$

\section{Spectral representation}

We represent $\psi$ and $\theta$ satisfying the boundary and symmetric conditions as infinite double series of orthogonal space functions in the form

$$
\psi=\sum_{l=0}^{\infty} \sum_{n=1}^{\infty} \hat{\psi}_{l, n} \sin (\operatorname{lax}) \sin (n z)
$$




$$
\theta=\sum_{l=0}^{\infty} \sum_{n=1}^{\infty} \hat{\theta}_{l, n} \cos (\operatorname{la} x) \sin (n z)
$$

where $l$ and $n$ are integers, $a$ is the horizontal wave number of the first mode $(l=n=1)$ and the coefficients $\hat{\psi}_{l, n}$ and $\hat{\theta}_{l, n}$ are functions of $\mathrm{R}$. The representations (3.1) and (3.2) transform the governing differential equations into the spectral domain of the spectra of the linear case.

Equations (3.1) and (3.2) can be written as

$$
\begin{aligned}
& \psi=\sum_{\gamma} \psi_{\gamma} S_{\gamma}, S_{\gamma}=-\operatorname{Exp} i(\operatorname{la} x+n z) \\
& \theta=\sum_{\gamma} \theta_{\gamma} C_{\gamma}, C_{\gamma}=-i \operatorname{Exp} i(\operatorname{la} x+n z)
\end{aligned}
$$

where $\sum_{\gamma}$ means summation over all integral lattice points in the ln-plane and $\gamma$ is a vector with components $(l, n)$. The orthogonality of $C_{\gamma}$ and $S_{\gamma}$ may be expressed in the form

$$
\int_{S} S_{\beta}^{*} S_{\alpha} \mathrm{d} S=\int_{S} C_{\beta}^{*} C_{\alpha} \mathrm{d} S=\delta_{\beta, \alpha}
$$

where $S$ is the surface $0 \leqslant x \leqslant 2 \pi / a,-\pi \leqslant z \leqslant \pi, \mathrm{d} S$ is the elementary area divided by the total area $4 \pi^{2} / a$ of the region, $S_{\beta}^{*}$ and $C_{\beta}^{*}$ are the complex conjugates of $S_{\beta}$ and $C_{\beta}$.

In order to determine the coefficients $\psi_{\gamma}$ and $\theta_{\gamma}$, we substitute (3.3) and (3.4) into (2.7) and (2.8) and obtain the following system of spectral equations

$$
\begin{aligned}
& \left(\alpha_{\gamma}^{4}+\sigma_{1}^{2} \alpha_{\gamma}^{2}\right)-l a \theta_{\gamma}=-\frac{a}{\operatorname{Pr}} B_{\gamma} \\
& \alpha_{\gamma}^{2} \theta_{\gamma}-\operatorname{laR} \psi_{\gamma}=-a H_{\gamma}
\end{aligned}
$$

where $\alpha_{\gamma}^{2}=l^{2} a^{2}+n^{2}, B_{\gamma}$ and $H_{\gamma}$ are respectively the vorticity and thermal advection spectra given by

$$
\begin{aligned}
& B_{\gamma}=-\sum_{\gamma_{1}} \sum_{\gamma_{2}}\left(l_{1} n_{2}-l_{2} n_{1}\right) \alpha_{\gamma}^{2} \psi_{\gamma_{1}} \psi_{\gamma_{2}} \\
& H_{\gamma}=\sum_{\gamma_{1}} \sum_{\gamma_{2}}\left(l_{1} n_{2}-l_{2} n_{1}\right) \psi_{\gamma_{1}} \theta_{\gamma_{2}}
\end{aligned}
$$

where the pairs $\gamma_{1}$ and $\gamma_{2}$ satisfy the selection rule

$$
\gamma=\gamma_{1}+\gamma_{2}, \quad \text { i.e. } l=l_{1}+l_{2}, \quad n=n_{1}+n_{2} .
$$

The nonlinear contributions can be more significantly expressed in terms of "coupling coefficients" defined by

$$
L_{\gamma, \beta, \alpha} \equiv \frac{1}{a} \int C_{\gamma}^{*} \frac{\partial\left(C_{\beta}, C_{\alpha}\right)}{\partial(x, z)} \mathrm{d} \sigma .
$$

Evaluation of these coupling coefficients by direct integration in (3.10) yields

$$
L_{\gamma, \beta, \alpha}=\left(l_{\beta} n_{\alpha}-l_{\alpha} n_{\beta}\right) \delta_{\gamma, \beta+\alpha}
$$


where $\delta_{\gamma, \beta+\alpha}$ is the Kronecker delta. The coupling coefficient vanishes unless the selection rule

$$
\gamma=\beta+\alpha
$$

holds; written in terms of the components, this means

$$
l_{\gamma}=l_{\beta}+l_{\alpha}, \quad n_{\gamma}=n_{\beta}+n_{\alpha}
$$

To find the solutions of (3.6) and (3.7), the temperature field $\theta(x, z)$ may be split into a mean temperature field $\bar{\theta}(z)$ and a deviation $\Theta(x, z)$ :

$$
\theta(x, z)=\bar{\theta}(z)+\Theta(x, z)
$$

where the mean temperature field $\bar{\theta}(z)$ is defined as the horizontal average over a full wave-length

$$
\bar{\theta}(z)=\frac{a}{2 \pi} \int_{0}^{2 \pi / a} \theta(x, z) \mathrm{d} x .
$$

This enables us to split (3.6) and (3.7) into the following set of equations for convenience:

$$
\left.\begin{array}{l}
\bar{\theta}_{\gamma}=\bar{H}_{\gamma} / n^{2} \quad \text { for } \quad l=0 \\
\alpha_{\gamma}^{2} \theta_{\gamma}-a l \mathrm{R} \psi_{\gamma}=-a H_{\gamma} \\
\left(\alpha_{\gamma}^{4}+\sigma_{1}^{2} \alpha_{\gamma}^{2}\right) \psi_{\gamma}=a l \theta_{\gamma}-\frac{a}{\operatorname{Pr}} B_{\gamma}
\end{array}\right\} \text { for } l \neq 0 .
$$

These equations are useful to obtain an expression for the modal Rayleigh number and in turn to study the interaction of different convective modes as explained in the next section.

\section{Modal Rayleigh number}

Elimination of $\theta_{\gamma}$ in (3.15) leads to

$$
\left(\mathrm{R}-\mathrm{R}_{\gamma}\right) \psi_{\gamma}=\frac{1}{l} H_{\gamma}+\frac{\alpha_{\gamma}^{2}}{a l^{2} \operatorname{Pr}} B_{\gamma}
$$

where

$$
\mathbf{R}_{\gamma}=\frac{\alpha_{\gamma}^{6}}{l^{2} a^{2}}+\frac{\sigma_{1}^{2} \alpha_{\gamma}^{4}}{l^{2} a^{2}}
$$

is called the Modal Rayleigh number. We note that as $\sigma^{2} \rightarrow 0$, this modal Rayleigh number $\mathbf{R}_{\gamma}$ tends to the one given by Kuo and Platzman [9] for the case of pure viscous flow. On the other hand, as $\sigma^{2} \rightarrow \infty$ (Darcy flow), $\mathrm{R}_{\gamma}$ tends to the one given by Rudraiah and Balachandra Rao [14] for the case of Darcy flow. In the linear theory $\left(H_{\gamma}=0, B_{\gamma}=0\right)$ a non-trivial solution of (4.1) requires $\mathrm{R}=\mathrm{R}_{\gamma}$. We write (3.9) in the form

$$
H_{\gamma}=\sum_{\beta} \sum_{\alpha} \bar{\theta}_{\beta} \psi_{\alpha} L_{\gamma, \beta, \alpha}+\sum_{\beta} \sum_{\alpha} \Theta_{\beta} \psi_{\alpha} L_{\gamma, \beta, \alpha}
$$


recognising the fact that $l_{\beta}=0$ in the first summation while $l_{\beta} \neq 0$ in the second one.

For a given mode $\gamma=(l, n), \mathrm{R}_{\gamma}$ is a continuous function of $a^{2}$ and in fact it is hyperbolic profile. The critical modal Rayleigh number $\left(\mathrm{R}_{\gamma}\right)_{c}$ and the corresponding critical wave number $a_{c}$ are given by

$$
\begin{aligned}
\left(\mathrm{R}_{\gamma}\right)_{c}= & \frac{1}{32 n^{2}}\left[3 n^{2}-\sigma_{1}^{2}+\sqrt{\left(\sigma_{1}^{2}+n^{2}\right)\left(\sigma_{1}^{2}+g n^{2}\right)}\right] \\
& \times\left[3 n^{2}+\sigma_{1}^{2}+\sqrt{\left(\sigma_{1}^{2}+n^{2}\right)\left(\sigma_{1}^{2}+9 n^{2}\right)}\right] \\
a_{c}^{2}=\frac{1}{4 l^{2}} & {\left[-\left(\sigma_{1}^{2}+n^{2}\right)+\sqrt{\left(\sigma_{1}^{2}+n^{2}\right)\left(\sigma_{1}^{2}+9 n^{2}\right)}\right] . }
\end{aligned}
$$

These are true for any mode consistant with our selection rule explained in Sec. 3. The minimum critical $R_{\gamma}$ and $a_{c}$ are obtained for the fundamental mode $(1,1)$ in the form

$$
\begin{aligned}
\left(\mathrm{R}_{\gamma}\right)_{c} \equiv & \left(\mathrm{R}_{11}\right)_{c}=\frac{1}{32}\left[3-\sigma_{1}^{2}+\sqrt{\left(1+\sigma_{1}^{2}\right)\left(9+\sigma_{1}^{2}\right)}\right]^{2} \\
& \times\left[3+\sigma_{1}^{2}+\sqrt{\left(\sigma_{1}^{2}+1\right)\left(\sigma_{1}^{2}+9\right)}\right] \\
a_{c}^{2}=\frac{1}{42} & {\left[\sqrt{\left(\sigma_{1}^{2}+1\right)\left(\sigma_{1}^{2}-9\right)-\sigma_{1}^{2}-1}\right] . }
\end{aligned}
$$

When $\sigma^{2} \rightarrow 0$, (4.6) gives $\mathrm{R}_{c}=6.75$ and $a_{c}^{2}=0.5$ which are the known values for the viscous flow given by Kuo and Platzman [9]. (Note that their $\mathbf{R}_{c}$ differs from our $\mathbf{R}_{c}$ by a factor $\pi^{4}$ because of our choice of the length scale.) Similarly, when $\sigma^{2}=10^{5}$, (4.6) gives $\mathrm{R}_{\mathrm{c}}=4 \sigma_{1}^{2}$ and $a_{c}=1$ which are the known values given by Lapwood [10]. To obtain the more general results, $\left(\mathrm{R}_{11}\right)_{c},\left(\mathrm{R}_{11} / \sigma^{2}\right)$ and $a_{c}$, given by (4.6) and (4.7), are numerically computed for different values of $\sigma^{2}$ and the results are given in Table 1.

Some interesting conclusions can be drawn from Table 1. For very small values of $\sigma^{2}$, the values of $\left(\mathrm{R}_{11}\right)_{c}$ and $a_{c}$ tend to those given by Kuo and Platzman [9] for the viscous flow whereas the values of $\left(R_{11}\right) d \sigma_{1}^{2}$ for those values of $\sigma^{2}$ are unrealistic. For large values of $\sigma^{2}\left(>10^{3}\right)$, the values of $\mathrm{R}_{11} / \sigma_{1}^{2}$ and $a_{c}$ tend to those given by Lapwood [10]. Recently,

Table 1.

Critical modal Rayleigh numbers and wave numbers for the fundamental mode

\begin{tabular}{llll}
\hline$\sigma^{2}$ & $a_{c}$ & $\left(\mathrm{R}_{11}\right)_{c} / \sigma_{1}^{2}$ & $\left(\mathrm{R}_{11}\right)_{c}$ \\
\hline $10^{0}$ & 0.71844 & 71.095907 & $7.2035218 \times 10^{0}$ \\
$10^{1}$ & 0.78686 & 11.000781 & $1.1146122 \times 10^{1}$ \\
$10^{2}$ & 0.93028 & 4.761219 & $4.8241236 \times 10^{1}$ \\
$10^{3}$ & 0.99054 & 4.078581 & $4.1324671 \times 10^{2}$ \\
$10^{4}$ & 0.99900 & 4.007891 & $4.0608431 \times 10^{3}$ \\
$10^{5}$ & 0.99962 & 4.000789 & $4.0536469 \times 10^{4}$ \\
\hline
\end{tabular}




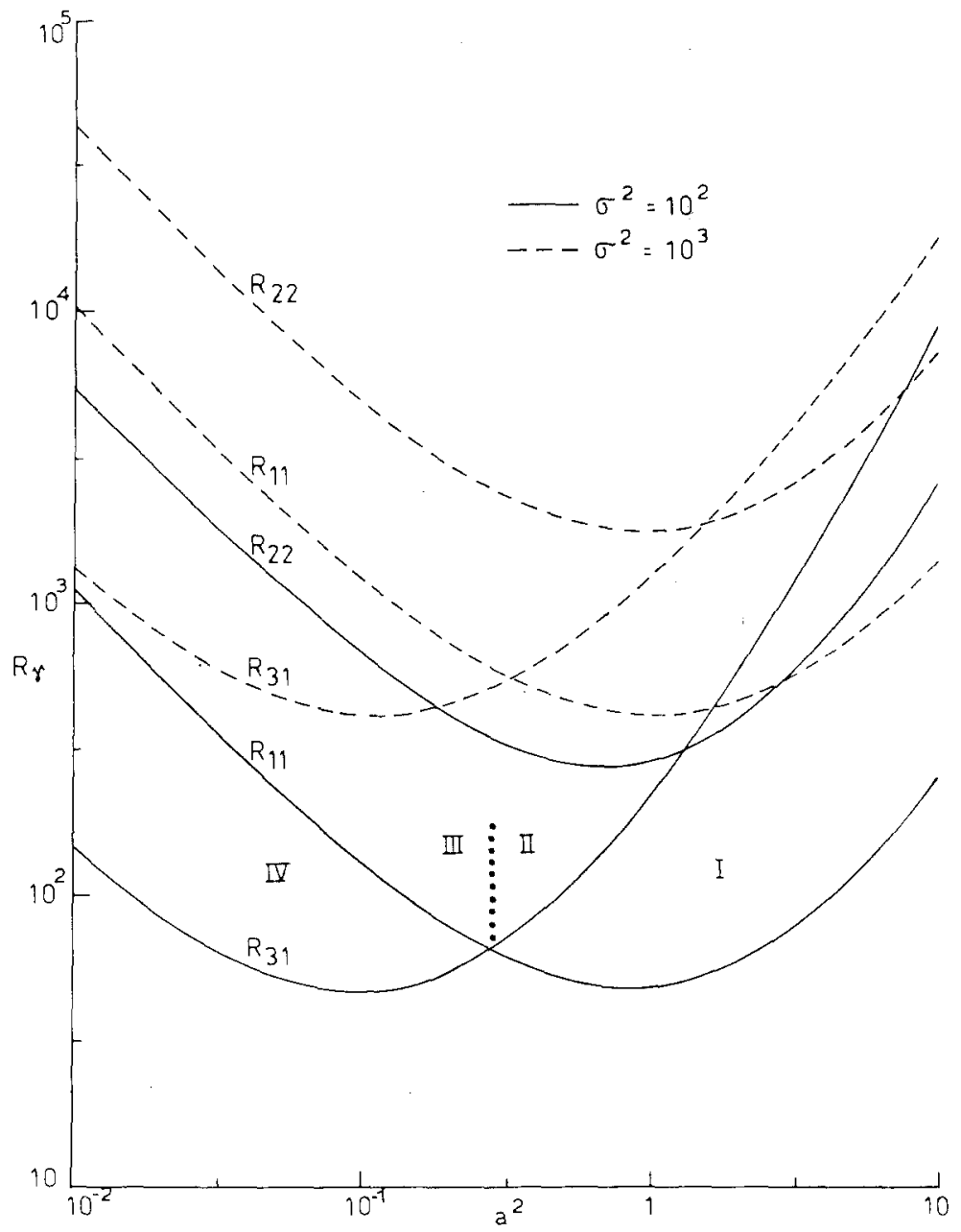

Figure 2. Modal Rayleigh number $\mathrm{R}_{\gamma}$ as a function of $a^{2}$

Rudraiah and Masuoka [15] have shown, using the method of matched asymptotic technique, that a transition zone (viz. transition from the Brinkman model to the Darcy model) exists for values of $\sigma^{2}$ in the range $10^{2}<\sigma^{2}<10^{3}$. The values of $\left(\mathrm{R}_{11}\right)_{c}$ and $a_{c}$ in this range are realistic and also predict the values in the transition zone. Therefore, for values of $\sigma^{2}$ up to $10^{3}$ we should use the usual definition of the Rayleigh number whereas for values of $\sigma^{2}>10^{3}$, the Lapwood Rayleight number $R_{11} / \sigma_{1}^{2}$ is more suitable. This aspect, emerging from the nonlinear theory, corroborates the results of Rudraiah and Masuoka [15] obtained from the linear theory. Thus, we can conclude that the Brinkman model, with a proper limiting process, gives the results of the viscous, transition and the Darcy flow regimes. The transition zone lies between the viscous and the Darcy flows as shown in Fig. 3. 
The variation of $\mathbf{R}_{\gamma}$ against $a^{2}$, for different modes $\gamma$, are computed and the results are shown in Fig. 2. This figure depicts the interactions of different modes for different values of $\sigma^{2}$. For instance, the curve representing the mode $(3,1)$ intersects that of the fundamental mode $(1,1)$ at $a^{2}=0.279$ for $\sigma^{2}=10^{2}$. Therefore, the fundamental mode is no longer a self-excited mode for the values of $a^{2}<0.279$ and hence rule out the possibility of the existence of steady solution in that region. In other words, subcritical instabilities are not possible in that region. Further, it is interesting to note that in the regions marked II and III in the neighbourhood of the line $a^{2}=0.279$ there are two self-excited modes for $\sigma^{2}=10^{2}$. Thus, a double-mode steady solution can exist in these regions in addition to the usual single-mode solution in which only the fundamental mode is the self-excited mode. In region I, a single-mode solution exists. A similar pattern of interaction repeats for higher values of $\sigma^{2}$. For example, for $\sigma^{2}=10^{3}$, the modes $(3,1)$ and $(1,1)$ intersect at $a^{2}=0.325$ (see Fig. 2). In other words, for values of $\sigma^{2}>10^{2}$, the point of intersection of the two modes $(3,1)$ and $(1,1)$ shift to the right of $a^{2}=0.279$, eventually approaching the Darcy result, $a^{2}=0.33$ (see [14]). In general, for a given $\sigma^{2}$, the point of intersection of the curves of the

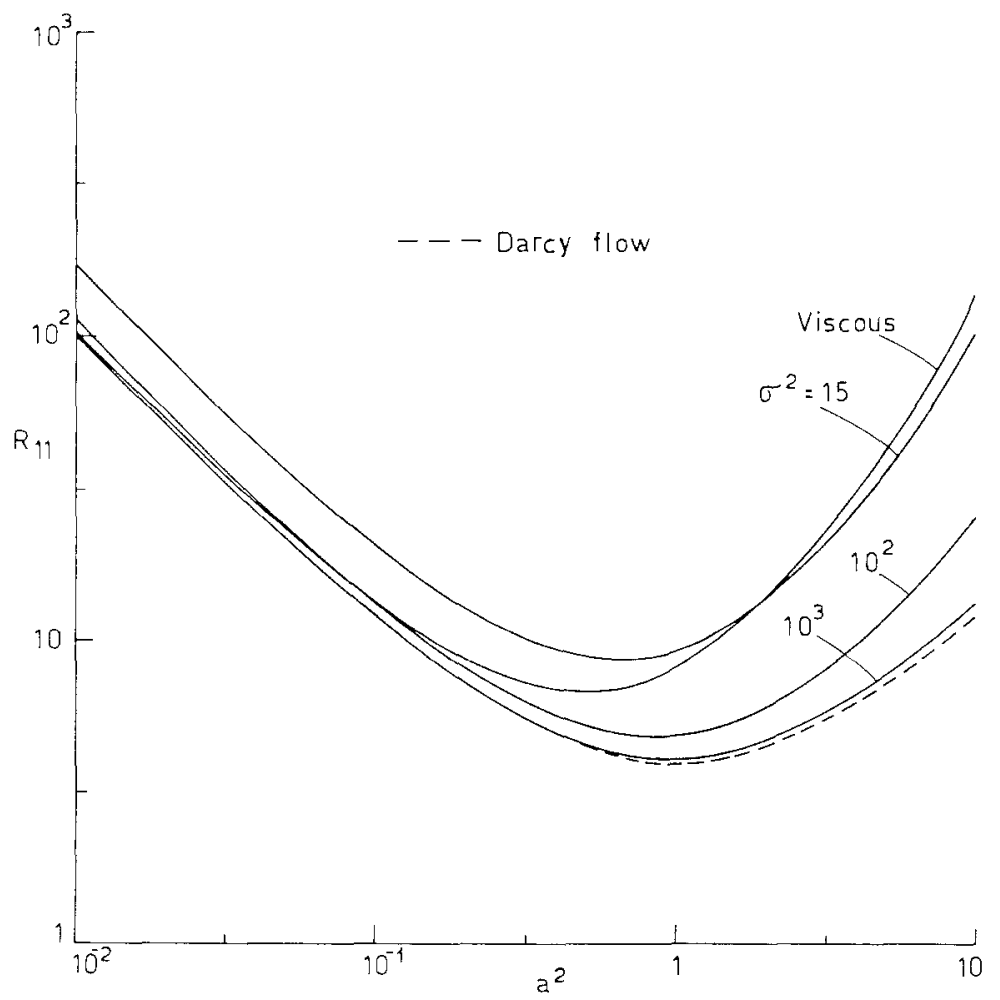

Figure 3. Variation of $R_{I 1}$ against $a^{2}$ for viscous, Darcy and Brinkman flows 
$(3,1)$ and $(1,1)$ modes is given by the solution of the cubic equation $720 x^{2}+\left(72 \sigma_{1}^{2}+216\right) x^{2}-8\left(\sigma_{1}^{2}+1\right)=0$

where $x=a^{2}$. In fact, this tends to the Darcy result, $x=1 / 3$ given by Rudraiah and Balachandra Rao [14] in the limit for large values of $\sigma^{2}$. The curves correspo.nding to the higher modes, $\gamma=(2,2)$ and $\gamma=(3,1)$, however, intersect at $a^{2}=1.3$ for $\sigma^{2}=10^{2}$ with $\mathrm{R}_{22}=\mathrm{R}_{31}=314.63$. For any $\sigma^{2}$, the point of intersection of the $(2,2)$ and $(3,1)$ modes is given by

$$
585 x^{3}+\left(45 \sigma_{1}^{2}-189\right) x^{2}-\left(54 \sigma_{1}^{2}+405\right) x-\left(35 \sigma_{1}^{2}+143\right)=0 .
$$

This also tends to the Darcy result $x=5 / 3$ for large values of $\sigma^{2}$. It is interesting to note that the modes $(2,2)$ and $(1,1)$ do not interact with one another, for the corresponding curves do not intersect. As one considers still higher modes, the intersection of these modes can be seen to be of an intricate cascade sort.

\section{Method of solution of the spectral equations}

The contribution of the nonlinear advection terms for the onset of convection is considered in this section. For this we need the solution of (4.1).

The method for solving (4.1) lies in developing each spectral element as an infinite series of the powers of a suitable parameter

$$
\Delta=\sqrt{\mathbf{R}-\mathbf{R}_{11}}
$$

which is a deviation from the critical Rayleigh number and usually measures the amplitude of the disturbance.

As in the Darcy model of Rudraiah and Balachandra Rao [14], we expand the spectral element $\psi_{\gamma}$ in the form

$$
\psi_{\gamma}=\psi_{\gamma, r} \Delta^{r}+\psi_{\gamma, \gamma+1} \Delta^{r+1}+\ldots
$$

where $r$ is the order of magnitude of an element $\psi_{\gamma}$ being the lowest power of $\Delta$ in the expansion. For example, since $\psi_{11}$ is, by definition, a first order element we can expand it in the form

$$
\psi_{11}=\psi_{111} \Delta+\psi_{113} \Delta^{3}+\ldots
$$

The exclusion of the odd-parity elements implies that the series expansion of a spectral element is in terms of only odd powers or only even powers of $\Delta$ according as the order of magnitude $r$ of the spectral element is odd or even. In the series representation of a spectral element $\psi_{\gamma}$, the coefficients of the type $\psi_{\text {lnp }}$ are all constants where $\psi_{\ln p}$ denotes the coefficients of $\Delta^{p}$ in the expansion of $\psi_{l n}$ (i.e. $\psi_{\gamma}$ ).

Now, expressing the spectral elements $\psi_{\gamma}, H_{\gamma}$ and $B_{\gamma}$ in (4.1) as power series in $\Delta$ using

$$
\left(\mathrm{R}-\mathrm{R}_{\gamma}\right)=-\left\{\left(\mathrm{R}-\mathrm{R}_{11}\right)-\Delta^{2}\right\}
$$


and equating the coefficients of $\Delta^{r}$, we get

$$
\left(\mathrm{R}_{\gamma}-\mathrm{R}_{11}\right) \psi_{\gamma, r}=\psi_{\gamma, r-2}+\frac{1}{l} H_{\gamma, r}+\frac{\alpha_{\gamma}^{2}}{a l^{2} \mathrm{Pr}} B_{\gamma, r}
$$

where $H_{\gamma, r}$ and $B_{\gamma, r}$ are expressed in terms of the flow spectrum using (3.8) and (3.9). In fact, as in the viscous case of Kuo and Platzman [9], (5.2) works as a recursion formula.

We obtain the following expressions for some of the important coefficients of the flow spectrum:

$$
\begin{aligned}
\psi_{111}= & \frac{1}{\sqrt{2}\left(1+a^{2}\right)} \\
\psi_{113}= & \frac{a^{2}\left(83+22 a^{2}+3 a^{4}\right)}{16 \sqrt{2}\left(a^{2}+1\right)^{3} A} \\
\psi_{133}= & \frac{a^{2}}{8 \sqrt{2}\left(a^{2}+1\right)^{3} A} \\
\psi_{224}= & \frac{2 a^{3}\left\{\operatorname{Pr}^{-1}\left(a^{2}+1\right)+\left(a^{2}+5\right)\right\}}{3\left(a^{2}+1\right)^{4}\left(5 a^{2}+5+\sigma_{1}^{2}\right) A} \\
\psi_{244}= & -\left[a^{3}\left\{\left(a^{2}+5\right)+\operatorname{Pr}^{-1}\left(a^{2}+4\right)\right\}\right] \\
& \times\left[( a ^ { 2 } + 1 ) ^ { 2 } A \left\{1023+765 a^{2}+189 a^{4}+15 a^{6}\right.\right. \\
& \left.\left.+3 \sigma_{1}^{2}\left(a^{4}+10 a^{2}+21\right)\right\}\right]^{-1}
\end{aligned}
$$

where

$$
A=91+10 \sigma_{1}^{2}+2\left(15+\sigma_{1}^{2}\right) a^{2}+3 a^{4} .
$$

Although the solution can be developed for arbitrary values of the cell-scale $a$, we use the critical wave number $a_{c}$ of the linear theory which varies with $\sigma^{2}$. Further, it is evident that the effect of the Prandtl number is seen in the fourth-order terms. The spectral coefficients, evaluated at $a=a_{c}$ for different values of $\sigma^{2}$ and $\mathrm{Pr}$, corresponding to mercury $(\operatorname{Pr}=0.025)$, air $(\operatorname{Pr}=0.687)$ and water $(\operatorname{Pr}=8)$ are shown in Table 2, which confirms the following:

(i) The value of $\psi_{111}$ and $\psi_{113}$, both of which contribute to the fundamental mode and hence to the spectral element $\psi_{11}$, decrease with the increase of $\sigma^{2}$ but are independent of Pr. A comparison of these with the values for the Darcy flow [14] reveals that $\psi_{111}$ in the Brinkman model differs from that of the Darcy flow whereas $\psi_{113}$ has the same qualitative behaviour in the two models.

(ii) The other third-order coefficient $\psi_{135}$ which contributes to the $\gamma=(1,3)$ made also decreases with the increasing $\sigma^{2}$ and is also independent of Pr. 


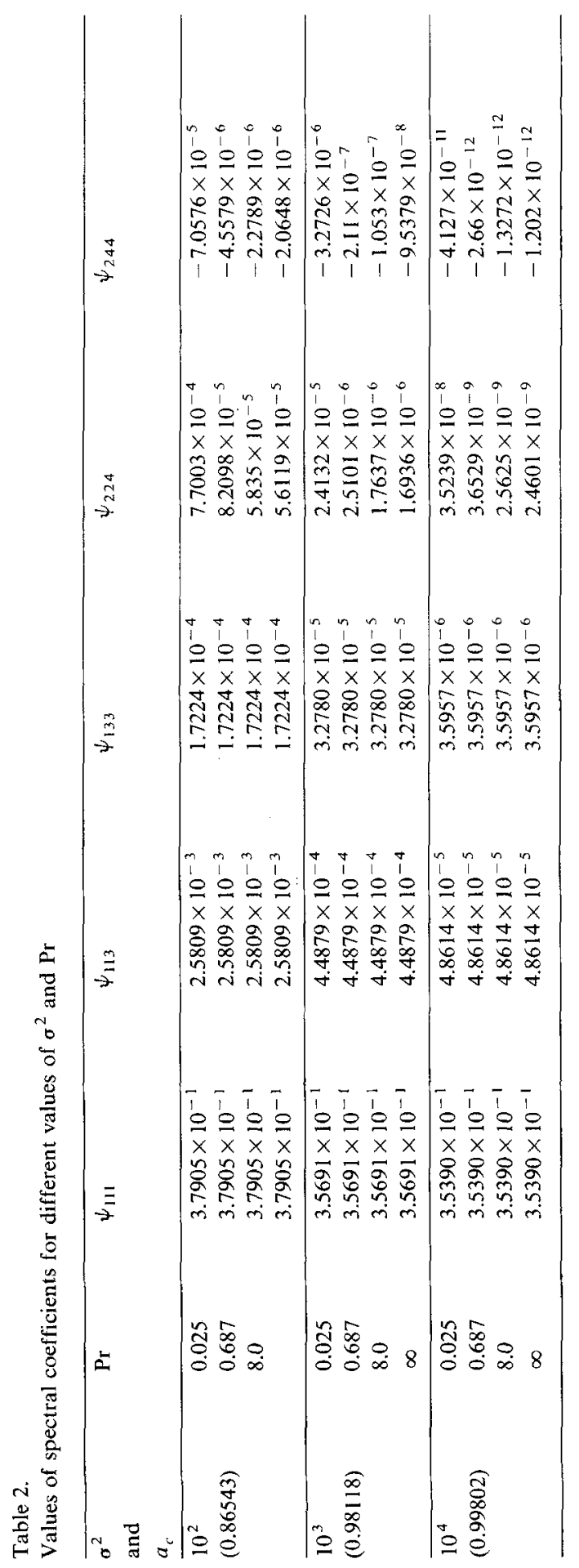


(iii) The contribution of $\operatorname{Pr}$ is seen only in the fourth order coefficients $\psi_{224}$ and $\psi_{244}$ which decrease numerically with increasing $\sigma^{2}$ and $\operatorname{Pr}$. For example, $\psi_{244}$ for water is nearly half of that for air for a given $\sigma^{2}$. A similar behaviour was observed in the case of the Darcy flow.

(iv) The effect of $\sigma^{2}$ is to decrease the spectral elements, for a given $\mathrm{Pr}$, thus damping the convective system.

(v) The effect of Pr on the fields is not so significant even as in the Darcy case discussed by Rudraiah and Srimani [16] and Rudraiah and Balachandra Rao [14].

\section{Spectral representation of the heat transport}

One of the objectives of the study of nonlinear convection is to determine the heat transport which depends on the imposed temperature difference between the boundaries. This is usually expressed as a functional relation between the Nusselt Number $\mathrm{Nu}$, and the Rayleigh number $\mathrm{R}$. The Nusselt number is the ratio of the actual heat transport rate to the rate at which heat would be transported by conduction alone. Thus,

$$
\mathrm{Nu}=-\frac{\left(\frac{\partial \bar{T}}{\partial z}\right)_{z=0}}{\Delta T / h}=-\frac{1}{\mathrm{R}}\left(\frac{\partial \bar{T}}{\partial z}\right)_{z=0} .
$$

Using (2.5) and (3.2), this takes the form

$$
\mathrm{Nu}=1-\frac{1}{\mathrm{R}} \sum_{\gamma} n_{\gamma} \vec{\theta}_{\gamma}
$$

where $\gamma=\left(0, n_{\gamma}\right)$ and $n_{\gamma}$ ranges over positive and negative even integers.

To determine $\mathrm{Nu}$, we expand the spectral elements in (6.2) in powers of the parameter $\Delta$ in the form

$$
\begin{aligned}
& \bar{\theta}_{02}=\bar{\theta}_{022} \Delta^{2}+\bar{\theta}_{024} \Delta^{4}+\bar{\theta}_{026} \Delta^{6}+\ldots \\
& \bar{\theta}_{04}=\bar{\theta}_{044} \Delta^{4}+\bar{\theta}_{046} \Delta^{6}+\ldots
\end{aligned}
$$

where $\bar{\theta}_{\text {onp }}$ is a constant being the coefficient of $\Delta^{p}$ in the power series expansion of the mean temperature spectral element $\bar{\theta}_{o n}$. Since $\bar{\theta}_{\gamma}$ appear in even powers of $\Delta$, it is more convenient to use a new parameter $\varepsilon$ defined by

$$
\varepsilon=\Delta^{2} / \mathrm{R}_{11}=\left(\mathrm{R}-\mathrm{R}_{11}\right) / \mathrm{R}_{11}
$$

so that

$$
\mathrm{R}=(1+\varepsilon) \mathrm{R}_{11}
$$

Now, the Nusselt number may be arranged as a power series in $\varepsilon$ :

$$
\mathrm{Nu}=N_{0}+N_{2} \varepsilon+N_{4} \varepsilon^{2}+\ldots
$$


where

$$
\begin{aligned}
& N_{0}=1, \\
& N_{2}=-4 \bar{\theta}_{022}, \\
& N_{4}=-N_{2}-4 R_{11}\left(\bar{\theta}_{024}+2 \bar{\theta}_{044}\right) .
\end{aligned}
$$

To obtain (6.6), we have substituted (6.4) for $R$ in (6.2) and expanded $(1+\varepsilon)^{-1}$ as a power series in $\varepsilon$. In other words, (6.6) is valid only for $\varepsilon<1$.

\subsection{Determination of the Nusselt number}

The spectrum $\vec{\theta}_{\gamma}$ corresponding to the disturbed horizontal mean temperature field is determined using (3.14). The expressions for some of these spectral coefficients are

$$
\begin{aligned}
& \vec{\theta}_{022}=-1 / 2 \\
& \bar{\theta}_{024}=-\frac{a^{2}}{16 A} \\
& \bar{\theta}_{044}=-\frac{a^{2}\left(a^{4}+10 a^{2}+41\right)}{16\left(a^{2}+1\right)^{2} A} .
\end{aligned}
$$

Substituting these in (6.6), we get

$$
N_{0}=1, \quad N_{2}=2, \quad N_{4}=\frac{3 a^{6}+a^{4}-135 a^{2}-645+3 \sigma_{1}^{2}\left(a^{2}+1\right)^{2}}{4 A} .
$$

Expressing $\mathrm{Nu}$ through terms of different orders, we can write

$$
\begin{aligned}
& \mathrm{Nu}^{(0)}=N_{0}=1 \\
& \mathrm{Nu}^{(2)}=N_{0}+N_{2} \varepsilon \\
& \mathrm{Nu}^{(4)}=N_{0}+N_{2} \varepsilon+N_{4} \varepsilon^{2} .
\end{aligned}
$$

The variation of $\mathrm{Nu}$ with respect to the Rayleigh number is shown in Fig. 4. In this figure, we have also compared our results with the experimental data of Combarnous and LeFur [4] and a good agreement is found for $\sigma^{2}=10^{4}$.

We know that the Nusselt number is function of the cell-scale $a$ as well as $R$. To the second order in $\Delta$ we have

$$
\begin{aligned}
& \mathrm{Nu}=\mathrm{Nu}^{(2)}+O\left(\varepsilon^{2}\right) \\
& \mathrm{Nu}^{(2)}=1+2 \varepsilon .
\end{aligned}
$$

Substituting $\varepsilon$ from (6.3), this becomes

$$
\mathrm{Nu}^{(2)}=\frac{2 \mathrm{R} a^{2}}{\left(a^{2}+1\right)^{2}\left(a^{2}+1+\sigma_{1}^{2}\right)}-1 .
$$




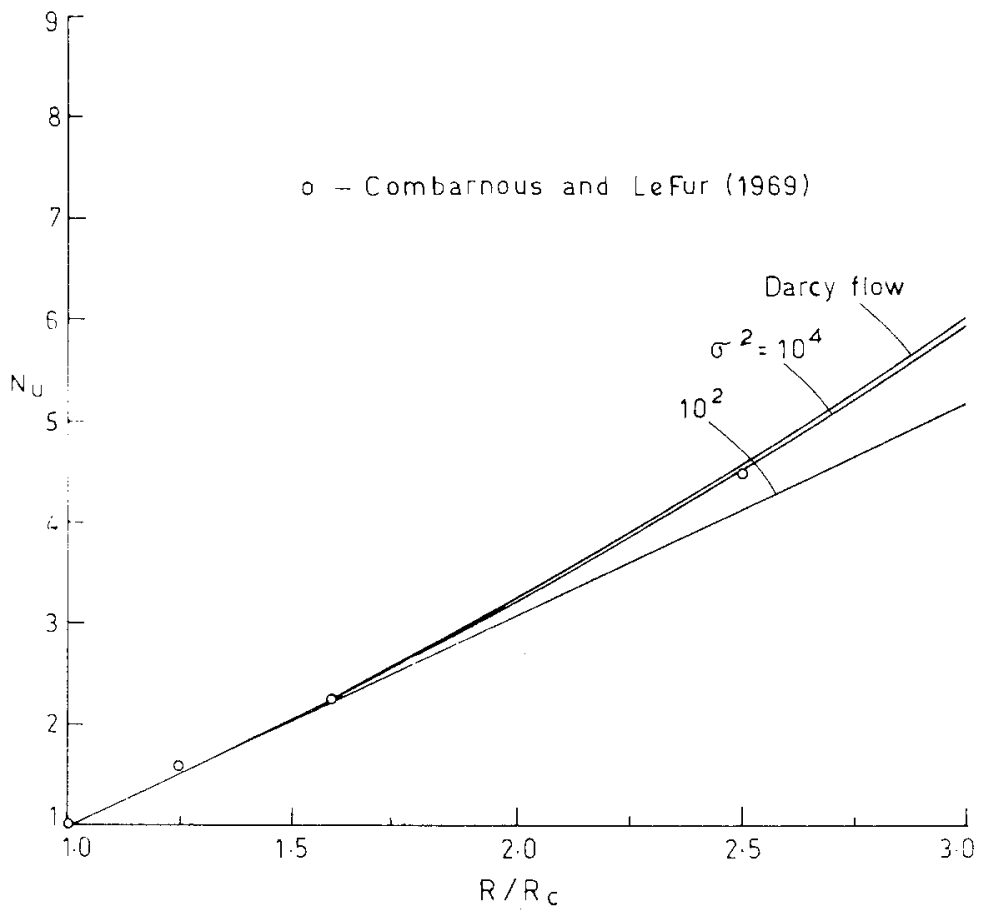

Figure 4. Variation of Nusselt number $\mathrm{Nu}$ with $\mathrm{R} / \mathrm{R}_{c}$

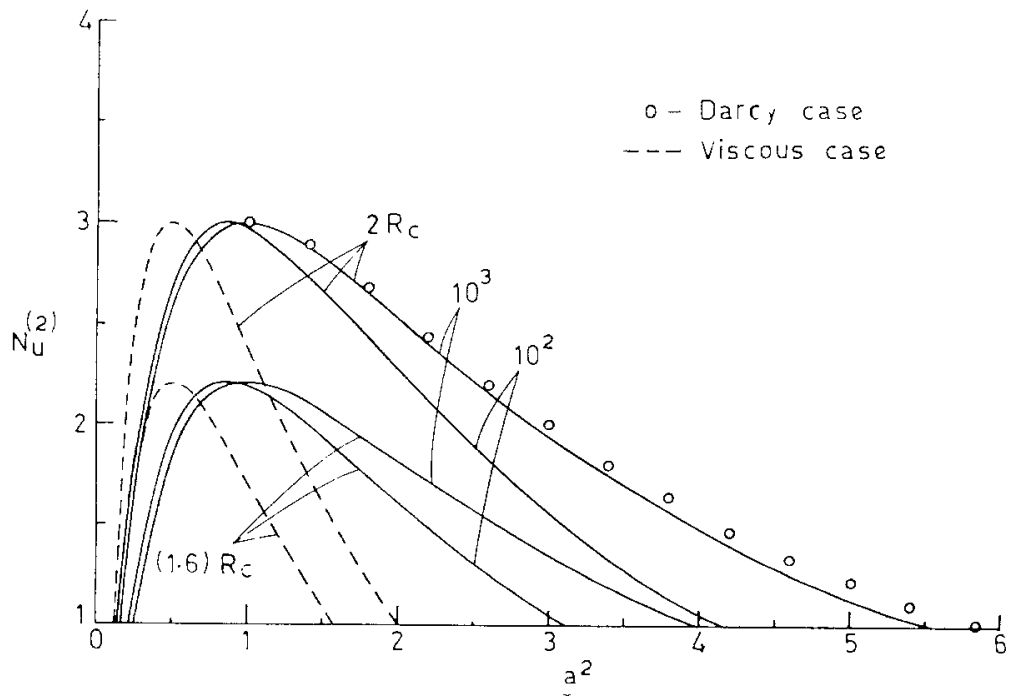

Figure 5. Variation of $\mathrm{Nu}^{(2)}$ against $a^{2}$ for $\mathrm{R}=1.6 \mathrm{R}_{c}, 2 \mathrm{R}_{c}$ 
Table 3.

Marginal slope of heat transport for different values of $\sigma^{2}$

\begin{tabular}{ll}
\hline$\sigma^{2}$ & Marginal slope \\
\hline $10^{4}$ & 0.49901 \\
$10^{3}$ & 0.49036 \\
$10^{2}$ & 0.42005 \\
\hline
\end{tabular}

Thus $\mathrm{Nu}^{(2)}$ computed for $\mathrm{R}=1.6 \mathrm{R}_{c}$ and $2 \mathrm{R}_{c}$ and for different values of $\sigma^{2}$ are represented in Fig. 5 as a function of $a^{2}$. In this figure, for the sake of comparison, the Darcy and viscous flow results are also shown. We observe that the second-order Nusselt number in the case of Darcy and viscous flows attains its maximum value for any fixed $\mathrm{R}$ when $a^{2}=1$ and $a^{2}=0.5$ respectively whereas in the case of Brinkman flow the maximum value of $\mathrm{Nu}^{(2)}$ varies with $\sigma^{2}$. For example, $\mathrm{Nu}^{(2)}$ is maximum at $a_{c}=0.98118$ for $\sigma^{2}=10^{3}, a_{c}=0.86543$ for $\sigma^{2}=10^{2}$ and $a_{c}=0.61915$ for $\sigma^{2}=10$. We see that for large values of $\sigma^{2}$ the results of Brinkman model tend to those of Darcy flow and for small values of $\sigma^{2}$ to those of the viscous flow. A similar behaviour is true also for $\mathrm{Nu}^{(4)}$. This proves that the results of the Brinkman model are more general and the other two extreme cases can be obtained with a proper limiting process.

The marginal slope of the heat transport function, using (6.5) is

$$
\left(\frac{\mathrm{d} \mathrm{Nu}^{(2)}}{\mathrm{dR}}\right)_{\mathrm{R}=\mathrm{R}_{c}}=\left(\frac{\mathrm{d} \mathrm{Nu}^{(4)}}{\mathrm{dR}}\right)_{\mathrm{R}=\mathrm{R}_{c}}=\frac{N_{2}}{\left(\mathrm{R}_{11}\right)_{c}} \text {. }
$$

In contrast to the marginal slope of value $1 / 2$ in the Darcy case, this marginal slope varies with $\sigma^{2}$. The marginal slope is computed for various values of $\sigma^{2}$ and the results are shown in Table 3.

From Table 3 it is clear that as $\sigma^{2}$ increases, the marginal slope of the heat transport tends to the well-known value of $1 / 2$ for the Darcy flow.

\section{Streamlines and mean temperature profiles}

In this section the streamlines and the mean temperature profiles are determined using the spectral coefficients for velocity and temperature discussed in Sec. 3.

\subsection{Streamline geometry}

The stream function $\psi$, given by (3.1), being a sine series in $x$ is antisymmetric with respect to the line $x=\pi / a$ and hence it is sufficient to consider only a half-cell. We, therefore, confine attention only to the 
region $0 \leqslant x \leqslant \pi / a, 0 \leqslant z \leqslant \pi$. For a given $\sigma^{2}$, the cell-scale $a$ is fixed and hence we get a rectangular region $D$ :

$$
D=\{(x, z) \mid 0 \leqslant x \leqslant \pi / a, 0 \leqslant z \leqslant \pi\} .
$$

In this rectangle, consider an arbitrary point $A(x, z)$ and let $B[\pi / a-x$, $\pi-z$ ] be the "image" of $A$. According to (3.3), we have

$$
\psi(A)=-\sum_{\gamma} \psi_{\gamma} \exp i(\operatorname{lax}+n z)
$$

where $\gamma=(l, n)$. Similarly,

$$
\begin{aligned}
\psi(B) & =-\sum_{\gamma} \psi_{\gamma} \exp i\left\{l a\left(\frac{\pi}{a}-x\right)+n(\pi-z)\right\} \\
& =-\sum_{\gamma} \psi_{\gamma}(-l)^{l+n} \exp \{-i(\operatorname{la} x+n z)\} .
\end{aligned}
$$

Since we consider only the even-parity components $(l, n),(l+n)$ is even so that

$$
\psi(B)=-\sum_{\gamma} \Psi_{\gamma} \exp \{-i(\operatorname{lax}+n z)\}
$$

Since $\psi_{-\gamma}=\psi_{\gamma}$, we have

$$
\psi(B)=\psi(A) \text {. }
$$

This means that the streamline pattern is radially symmetric with respect to the centre of the region $D$.

Expanding $\psi$, given by (3.4), in terms of powers of $\Delta$, we get

$$
\begin{aligned}
\psi= & 4\left(\psi_{11} \sin z+\psi_{13} \sin 3 z\right) \sin a x \\
& +4\left(\psi_{22} \sin 2 z+\psi_{24} \sin 4 z\right) \sin 2 a x+\ldots
\end{aligned}
$$

The streamlines corresponding to $\Delta=1$ are computed for different values of $\sigma^{2}$ and are plotted in Fig. 6 . For the sake of comparison, the viscous $\left(\sigma^{2} \rightarrow 0\right)$ and the Darcy $\left(\sigma^{2} \rightarrow \infty\right)$ cases are also shown in this figure. From this it is clear that the streamline pattern $\psi=1$ is circular in the case of the Darcy flow and highly elliptical in the viscous case while it gets deformed into slightly elliptical pattern in the transition region given by the Brinkman flow. When the temperature difference is further increased to $\Delta=2$, the streamline pattern $\psi=1$ tends to a rectangle as shown in Fig. 7, thus pushing the volume transport closer to the boundaries of the cell.

\subsection{Mean temperature distribution}

Besides the heat transport reported in Sec. 6.1, the next important quantity obtainable from the nonlinear solution is the modified mean temperature distribution which is discussed in this section. 


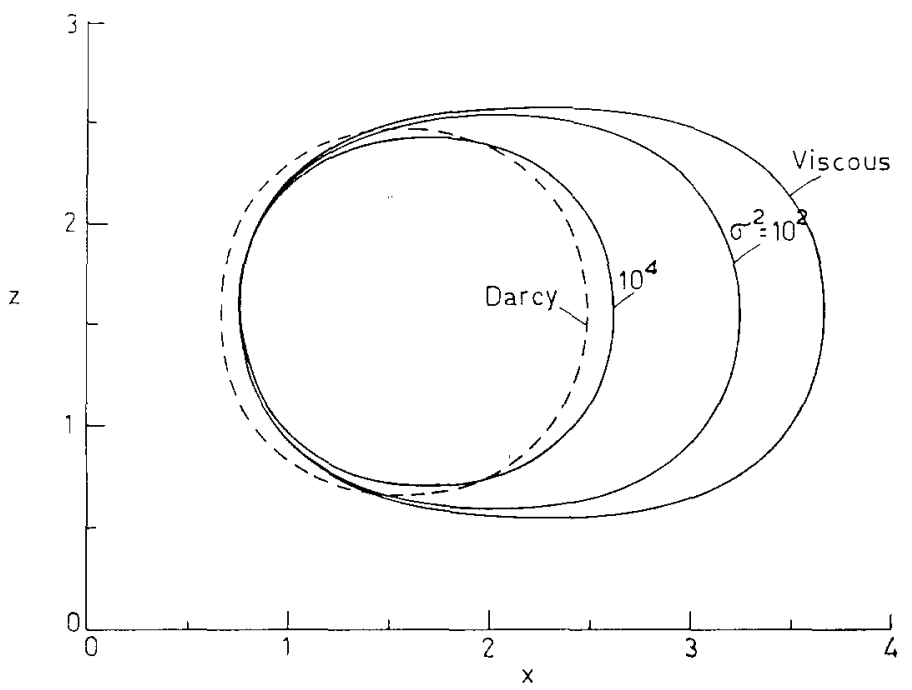

Figure 6. Streamline $\psi=1$ for $\Delta=1$

Now, considering the horizontally averaged temperature using (2.5), (3.2) and (3.13), we get

$$
\bar{\theta}=-z+\frac{2}{\mathrm{R}}\left[\left(\bar{\theta}_{022} \Delta^{2}+\bar{\theta}_{024} \Delta^{4}\right) \sin 2 \pi z+\left(\bar{\theta}_{044} \Delta^{4}\right) \sin 4 \pi z\right] .
$$

The mean temperature profiles for different values of $\sigma^{2}$ are shown in Fig. 8 for $\Delta=1$ to depict the effect of permeability. Further, to know the

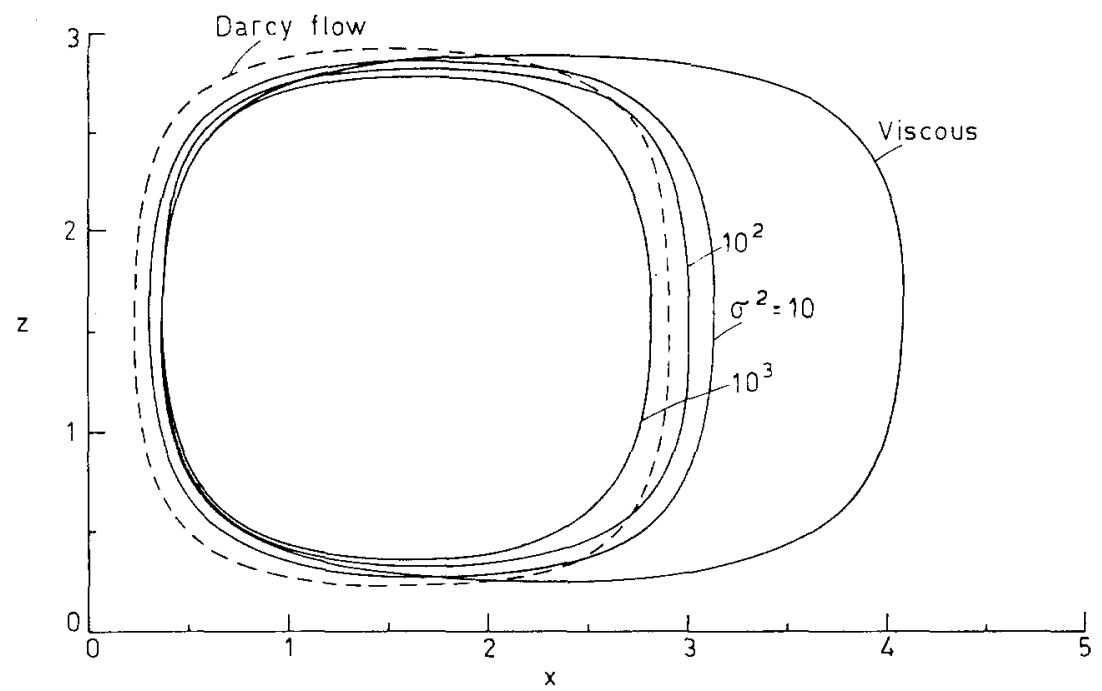

Figure 7. Streamline $\psi=1$ for $\Delta=1$ 


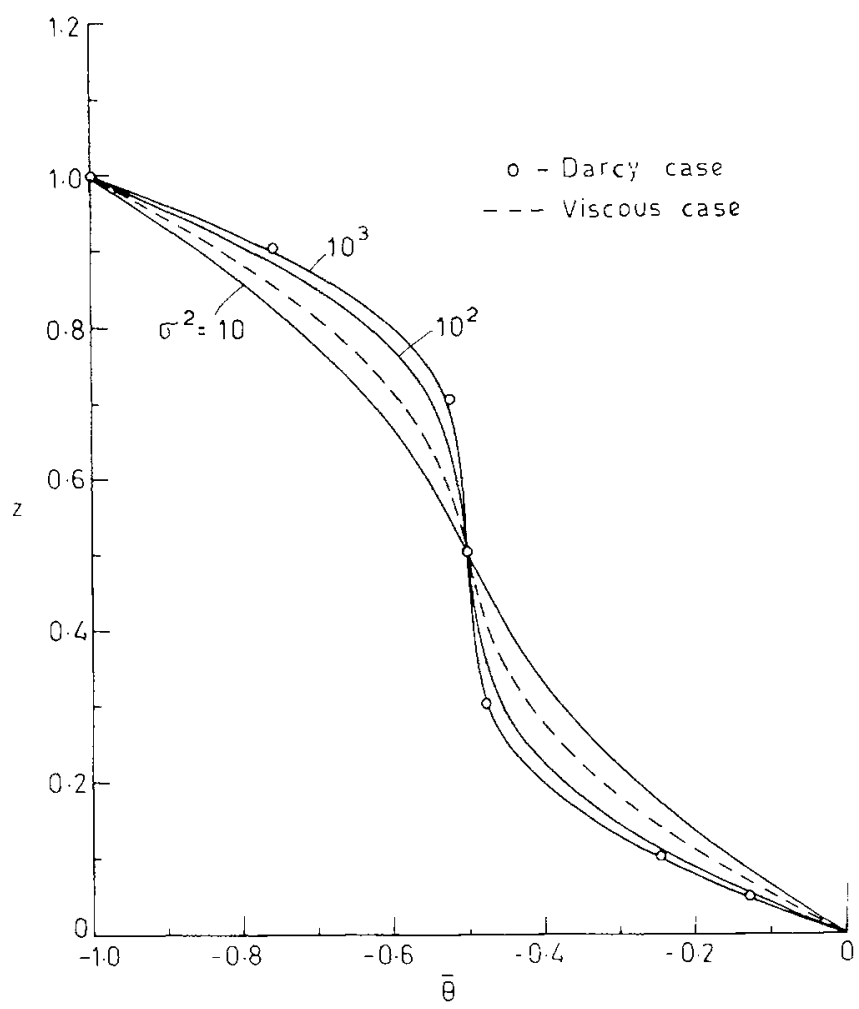

Figure 8. Comparison of the mean temperature distribution $\bar{\theta}$ for different $\sigma^{2}$ for $\Delta=1$

distortion in the mean temperature profile due to the increase in the temperature difference, it is computed for different values of $\Delta$ and the results are shown in Fig. 9 for $\sigma^{2}=10^{2}$ and in Fig. 10 for $\sigma^{2}=10^{3}$. Each of these profiles has a point of inflexion at $z=0.5$, midway between the boundaries. In particular, we note that although there exists only one point of inflexion $z=0.5$, common to all the profiles, for smaller values of $\Delta$ viz., $\Delta=0.5$ and 0.8 there exist two more points of inflexion for higher values of $\Delta$ at the points given by the solution of

$$
\cos 2 \pi z=-\frac{\bar{\theta}_{02}}{8 \bar{\theta}_{04}} .
$$

These curves also show clearly the effects of convective heat transfer and permeability of the medium on the mean temperature distribution. The most striking feature of these effects is that around $\Delta=1$ a region of isothermal stratification is produced by convection in the middle of the layer. In other words, there is a reversal in the mean temperature profile at the middle of the layer. We note that as $\Delta$ increases, say for $\Delta>2$, the 


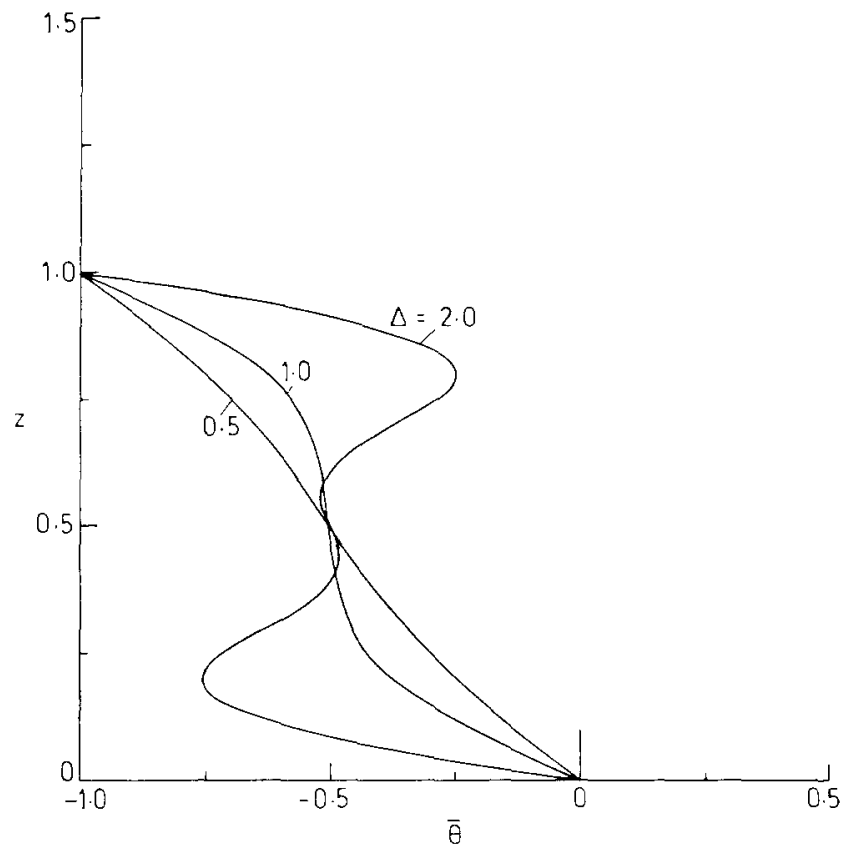

Figure 9. Mean temperature distribution $\bar{\theta}$ for $\sigma^{2}=10^{2}$

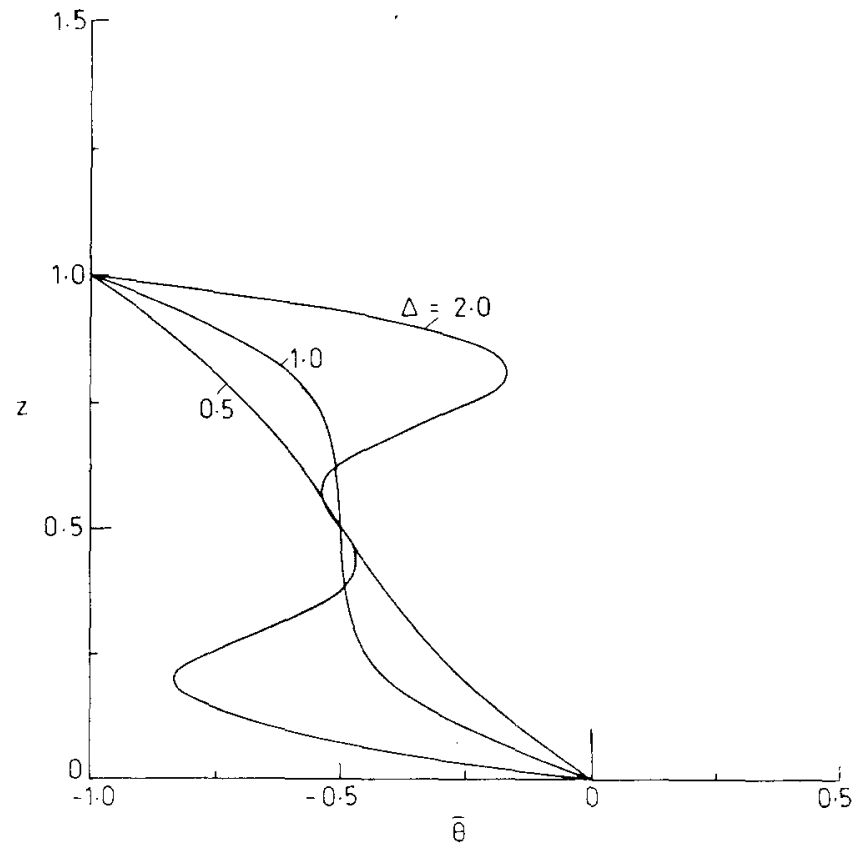

Figure 10. Mean temperature distribution $\bar{\theta}$ for $a^{2}=10^{3}$ 
mean temperature profiles get distorted, possibly due to the limitation of our solution.

\section{Discussion and conclusion}

One of the most striking features of the results presented in the earlier sections is that the Brinkman model generalizes the study of convection in a porous medium in the sense that for small and large values of the permeability parameter $\sigma^{2}$ the results approach respectively to those of the viscous and the Darcy flows in addition to providing results in the transition zone (i.e. $10^{2}<\sigma^{2}<10^{3}$ ). The Brinkman model considered in this paper also overcomes the mathematical difficulty inherent in the Darcy model.

The results obtained here using the spectral analysis technique agree well, for large values of $\sigma^{2}$, with those of Rudraiah and Srimani [16] obtained using the power integral technique. The novelty of using the spectral analysis technique is that it is elegant and takes care of cross-interactions of higher convective modes. The possibility of the existence of steady solution with two self-excited modes in certain regions is predicted in Fig. 2, which fact could not be obtained by the power integral technique. The results on temperature modes, shown in Figs. 8-10, reveal that there is a reversal in the horizontally averaged temperature profiles at the middle of the layer with an isothermal stratification around $\Delta=1$. However, for $\Delta>2$, distortions occur which are possibly due to the limitation of the solution obtained here.

A comparison of our results with those of the viscous flow [9] shows that the solution of the heat transport converges must faster in the case of porous medium. In fact, the percentage of deviation of the second-order Nusselt number is only 2.55 percent for $\sigma^{2}=10^{3}$ at $\Delta=2$. In contrast to the degeneracy of $\mathrm{Nu}^{(4)}$ of the viscous flow, in the present porous case $\mathrm{Nu}^{(4)}$ has a summative effect. Our results on heat transport are in good agreement with the experimental data of Combarnous and Le Fur [4]. The marginal slope of $\mathrm{Nu}-\mathrm{R}$ relation increases with $\sigma^{2}$ and tends to 0.5 , which is the exact value for the Darcy flow. In particular, it is shown in Fig. 5, that $\mathrm{Nu}$ attains the maximum at the critical wave number obtained from the linear theory corresponding to different values of $\sigma^{2}$.

The stream function given by (3.1) is evaluated for different values of $\sigma^{2}$ when $\Delta=1$ and the profiles are shown in Fig. 6 . The streamline shrinks from a highly elliptical pattern of the viscous case to a circular pattern for the Darcy flow through a transitional pattern which is slightly elliptical for $\sigma^{2}=10^{2}$. When $\Delta=2$, the streamline pattern which is a square for the Darcy flow tends to be rectangular, shown in Fig. 7, as $\sigma^{2}$ decreases thus pushing the volume transport closer to the boundary of the cell. 
Finally, we conclude that the inductive procedure adopted in the recursion formula (5.2) to evaluate the spectral coefficients fails to yield the solution when $R_{\gamma}=R_{11}$ for certain values of $a^{2}$. This results in the degeneracy of the solution. Using the definition of $R_{\gamma}$ given in (4.2), we find that the values of $a^{2}$ which result in the degeneracy of the solution of (5.2) are given, for different modes $\gamma=(l, n)$, by the solution of the cubic equation

$$
\left(l^{2} x+n^{2}\right)^{3}-l^{2}(x+1)^{3}+\sigma_{1}^{2}\left\{\left(l^{2} x+n^{2}\right)^{2}-l^{2}(x+1)^{2}\right\}=0
$$

where $x=a^{2}$. Corresponding to these points of degeneracy, a steady solution may exist in which there are two self-excited modes. At such exceptional points the method developed for the solution of (5.2) breaks down.

\section{Acknowledgements}

This work is sponsored by the University Grants Commission of India under the DSA programme.

The research by one of the authors (S.B.R) was supported by the UGC under the Faculty Improvement Programme.

\section{References}

1. Beavers GS and Joseph DD (1967) Boundary conditions at a naturally permeable wall. J Fluid Mech 30:197

2. Brenner H (1970) Rheology of two-phase systems. Ann Rev Fluid Mech 2:137

3. Brinkman HC 1947 Calculation of the viscous force exerted by a flow in fluid on a dense swarm of particles. Appl Sci Res A1:27

4. Combarnous $M$ and Le Fur B (1969) Transfert de chaleur par convection naturalle dans une couche poreuse horizontale. Comptes Rendus 269:1009

5. Elder JW (1967) Steady free convection in a porous medium heated from below. J Fluid Mech 27:29

6. Friedrich R and Rudraiah N (1981) Numerical Study of Large Amplitude Convection in a Rotating Fluid Saturated Porous Lyaer. Accepted for presentation at the Fourth GAMM-Conference on Numerical Methods in Fluid Mechanics, Oct. 7-9, 1981 at ENSTA (Paris)

7. Horton CW and Rogers FT (1945) Convection currents in a porous medium. J Appl Phys 16:367

8. Katto $Y$ and Masuoka T (1967) Criterion for the onset of convective flow in a fluid in porous medium. Int $\mathbf{J}$ Heat Mass Transfer 10:297

9. Kuo HL and Platzman GW (1961) A normal mode nonlinear solution of the Rayleigh convection problem. Bet Phys Frei Atmos Ed 33:\$137

10. Lapwood ER (1948) Convection of a fluid in a porous medium. Proc Camb Phil Soc 44:508

11. Malkus WVR and Veronis G (1958) Finite-amplitude cellular convection. J Fluid Mech $4: 225$ 
12. Morales M, Spinn CW and Smith JM (1951) Velocities and effective thermal conductivities in packed beds. Ind Engg Chem 43:225

13. Palm E, Weber JE and Kvenvold $O$ (1972) On steady convection in a porous medium. J Fluid Mech 54:153

14. Rudraiah $N$ and Balachandra Rao $S$ (1982) Nonlinear cellular convection and heat transport in a porous medium. Appl Sci Res 39:21

15. Rudraiah $\mathrm{N}$ and Masuoka $\mathrm{T}$ (1982) Asymptotic analysis of natural convection through horizontal porous layer. Int J Engg Sci 20:27

16. Rudraiah N and Srimani PK (1980) Finite-amplitude cellular convection in a fluid saturated porous layer. Proc R Soc, Lond A373:199

17. Rudraiah $N$ and Veerabhadraiah $R$ (1977) Temperature distribution in Couette flow past a permeable bed. Proc Indian Acad Sci 86A:6, 537

18. Rudraiah $\mathrm{N}$ and Veerabhadraiah R (1978) Effects of buoyancy on the free surface flow past a permeable bed. Wärme-und Stoffübertragung 11:265

19. Rudraiah $\mathrm{N}$ and Veerabhadraiah $\mathrm{R}$ (1979) Buoyancy effects on the plane couette flow past a permeable bed. J Math Phys Sci, India 13:6, 523

20. Rudraiah N, Veerappa B and Balachandra Rao S (1980) Effects of nonuniform thermal gradient and adiabatic boundaries on convection in porous media. ASME $J$ Heat Transfer 102:254

21. Ruth DW (1980A) Model for convection in horizontal porous media layers. AIAA 18th Aerospace Sciences Meeting AIAA-80-0090.

22. Tam CKW (1969) The drag on a cloud of spherical particles in low Reynolds number flow. J Fluid Mech 38:537

23. Taylor GI (1971) A model for the boundary conditions of a porous material. Part I, J Fluid Mech 49:319

24. Straus JM (1974) Large amplitude convection in porous media. J Fluid Mech 64:51 